This item was submitted to Loughborough's Research Repository by the author.

Items in Figshare are protected by copyright, with all rights reserved, unless otherwise indicated.

\title{
Molecular dynamics simulations of oxide memristors: crystal field effects
}

PLEASE CITE THE PUBLISHED VERSION

http://dx.doi.org/10.1063/1.3622665

\section{PUBLISHER}

(c) American Institute of Physics

VERSION

VoR (Version of Record)

LICENCE

CC BY-NC-ND 4.0

REPOSITORY RECORD

Savel'ev, Sergey, A.S. Alexandrov, A.M. Bratkovsky, and R.S. Williams. 2019. "Molecular Dynamics Simulations of Oxide Memristors: Crystal Field Effects". figshare. https://hdl.handle.net/2134/12718. 


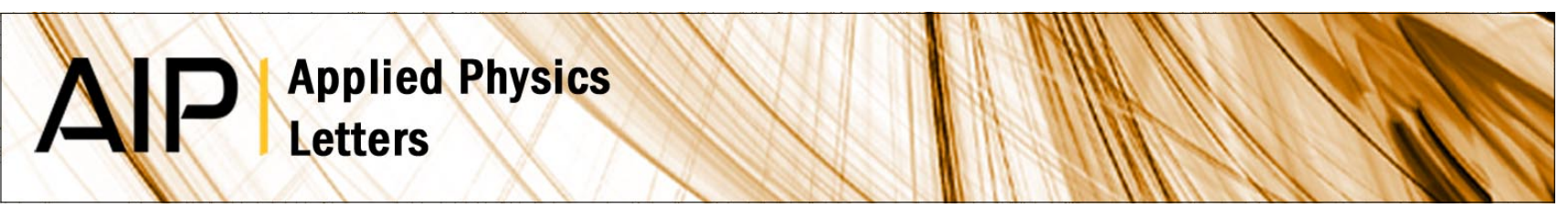

\section{Molecular dynamics simulations of oxide memristors: Crystal field effects}

S. E. Savel'ev, A. S. Alexandrov, A. M. Bratkovsky, and R. Stanley Williams

Citation: Appl. Phys. Lett. 99, 053108 (2011); doi: 10.1063/1.3622665

View online: http://dx.doi.org/10.1063/1.3622665

View Table of Contents: http://apl.aip.org/resource/1/APPLAB/v99/i5

Published by the AIP Publishing LLC.

Additional information on Appl. Phys. Lett.

Journal Homepage: http://apl.aip.org/

Journal Information: http://apl.aip.org/about/about_the_journal

Top downloads: http://apl.aip.org/features/most_downloaded

Information for Authors: http://apl.aip.org/authors

\section{ADVERTISEMENT}
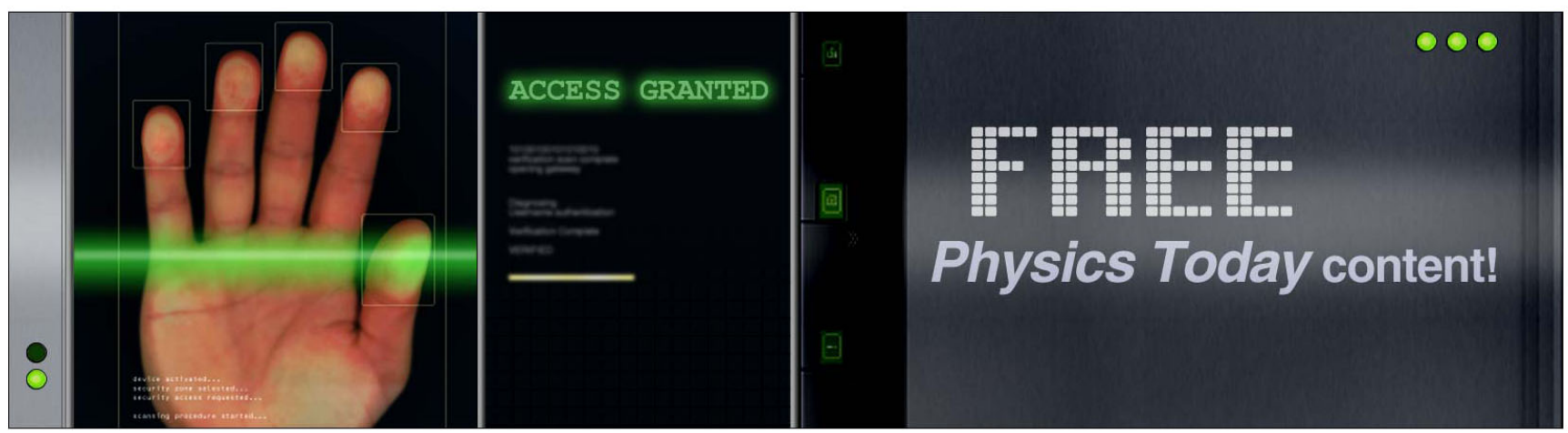


\title{
Molecular dynamics simulations of oxide memristors: Crystal field effects
}

\author{
S. E. Savel'ev, ${ }^{1}$ A. S. Alexandrov, ${ }^{1,2, a)}$ A. M. Bratkovsky, ${ }^{2}$ and R. Stanley Williams ${ }^{2}$ \\ ${ }^{1}$ Department of Physics, Loughborough University, Loughborough LE11 3TU, United Kingdom \\ ${ }^{2}$ Hewlett-Packard Laboratories, 1501 Page Mill Road, Palo Alto, California 94304, USA
}

(Received 19 May 2011; accepted 9 July 2011; published online 3 August 2011)

\begin{abstract}
We present molecular-dynamic simulations of memory resistors (memristors) including the crystal field effects on mobile ionic species such as oxygen vacancies appearing during operation of the device. Vacancy distributions show different patterns depending on the ratio of a spatial period of the crystal field to a characteristic radius of the vacancy-vacancy interaction. There are signatures of the orientational order and of spatial voids in the vacancy distributions for some crystal field potentials. The crystal field stabilizes the patterns after they are formed, resulting in a non-volatile switching of the simulated devices. (C) 2011 American Institute of Physics. [doi:10.1063/1.3622665]
\end{abstract}

Electrical switching in thin-film oxide nanodevices attracts renewed attention potentially enabling scaling of logic and memory circuits well beyond the current state of the art techniques. ${ }^{1}$ The microscopic nature of resistance switching and a charge transport in such devices are still a subject of debate, but there is growing evidence that nonvolatile switching requires some sort of atomic rearrangement of mobile ionic species that drastically affects the current. The microscopic understanding of the atomic-scale mechanism and identification of the material changes within the device appear to be invaluable for controlling and improving the memristor performance.

The number of oxygen vacancies within the volume $10 \times 10 \times 2 \mathrm{~nm}^{3}$ of perspective nano-memristors, such as based on an amorphous layer of titanium dioxide, $\mathrm{TiO}_{2-x}$, could be as small as about a thousand, so that the conventional statistical (diffusion) approach for dealing with manyparticle systems may fail. Recently, we have proposed a model for the kinetic behavior of oxide memristors and studied it using the molecular dynamics (MD) simulations of the Langevin equations describing the thermal diffusion and drift of interacting oxygen vacancies. ${ }^{2}$ Our MD simulations revealed a significant departure of the vacancy distributions across the device from that expected within a standard driftdiffusion approximation. The channel formation in systems like $\mathrm{TiO}_{2}, \mathrm{NiO}, \mathrm{VO}_{2}$ is certainly accompanied by local heating that is witnessed by the local emergence of high-temperature phases and observed by thermal microscopy and other techniques. ${ }^{3-5}$ Accounting for local heating, we extended our MD modeling of oxygen vacancies driven by an external bias voltage by taking into account temperature gradients in thin films of oxide memristors. ${ }^{2}$ Temperature gradients, producing strong variations of the rate of diffusion, affect the vacancy patterns in memristors. Our simulations indicated that variations of temperature across the memristor favor the formation of short-circuiting or shunting vacancy channels. At the same time, considerable temperature gradients along the sample can, by contrast, produce vacancy-poor regions where vacancy shunts are not formed and electron percolation paths are blocked. Here, we extend our MD simulations

\footnotetext{
${ }^{\text {a) }}$ Author to whom correspondence should be addressed. Electronic mail: A.S.Alexandrov@lboro.ac.uk.
}

of the memristor including the crystal field in the Langevin equations.

Observations of oxygen vacancy migration and clustering in bulk ${ }^{6}$ and nanoscale ${ }^{3,7}$ samples of $\mathrm{TiO}_{2}$, induced by an electric-field, allow us to model a memristor ${ }^{2}$ with the vacancy interaction potential, Fig. 1, with the results shown in Fig. 2. In the model, there is a reduced rutile thin layer $\mathrm{TiO}_{2-x}$ near one of the metallic electrodes stabilized by the Coulomb mirror potential. Vacancies from this layer can drift toward the opposite electrode pushed by a pulse of an electric field. Vacancies interact with each other via the pairwise potential $W$ and with the electric field corresponding to the time-dependent deterministic force $F$. The environment exerts an independent Gaussian random force, $\vec{\xi}$, on each particle with zero mean and intensity controlled by the temperature. Different from our previous studies, ${ }^{2}$ we now include the periodic crystal field potential $U\left(\boldsymbol{x}_{i}\right)$ in the overdamped Langevin equations describing the drift-diffusion of the ith particle as

$$
\begin{aligned}
\eta \frac{d \boldsymbol{x}_{i}^{\alpha}}{d t}= & F_{i}^{\alpha}\left(\boldsymbol{x}_{i}, \mathrm{t}\right)-\sum_{i} \frac{\partial U\left(\boldsymbol{x}_{i}\right)}{\partial x_{i}^{\alpha}}-\sum_{j \neq i} \frac{\partial W\left(\boldsymbol{x}_{i}-\boldsymbol{x}_{j}\right)}{\partial x_{i}^{\alpha}} \\
& +\sqrt{2 k_{B} T \eta} \xi_{i}^{\alpha}(t) .
\end{aligned}
$$

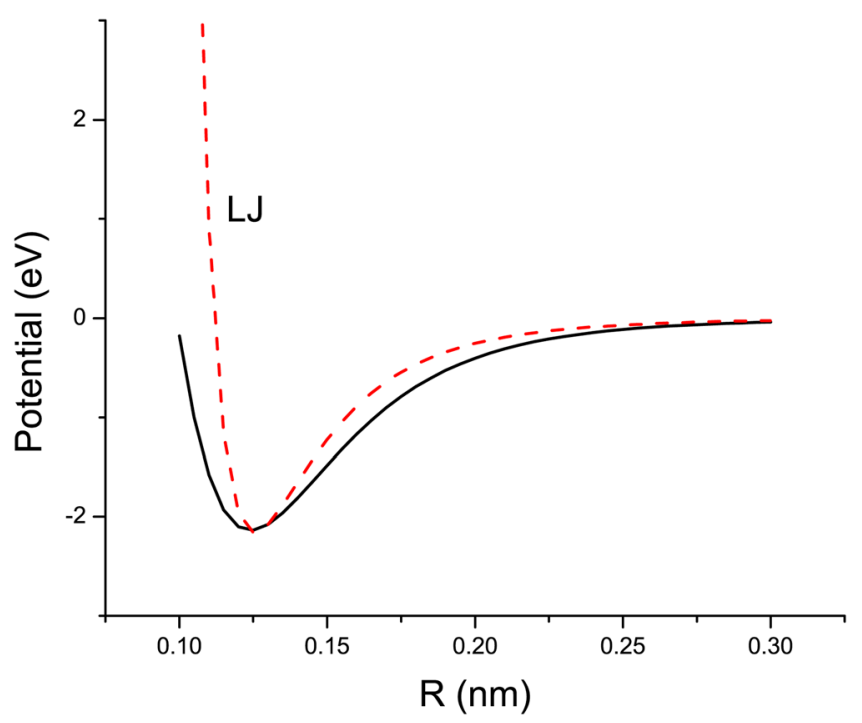

FIG. 1. (Color online) The Lennard-Jones potential (steeper curve) compared with the $\mathrm{O}^{2-}$ vacancy-vacancy interaction potential, Eq. (2). 


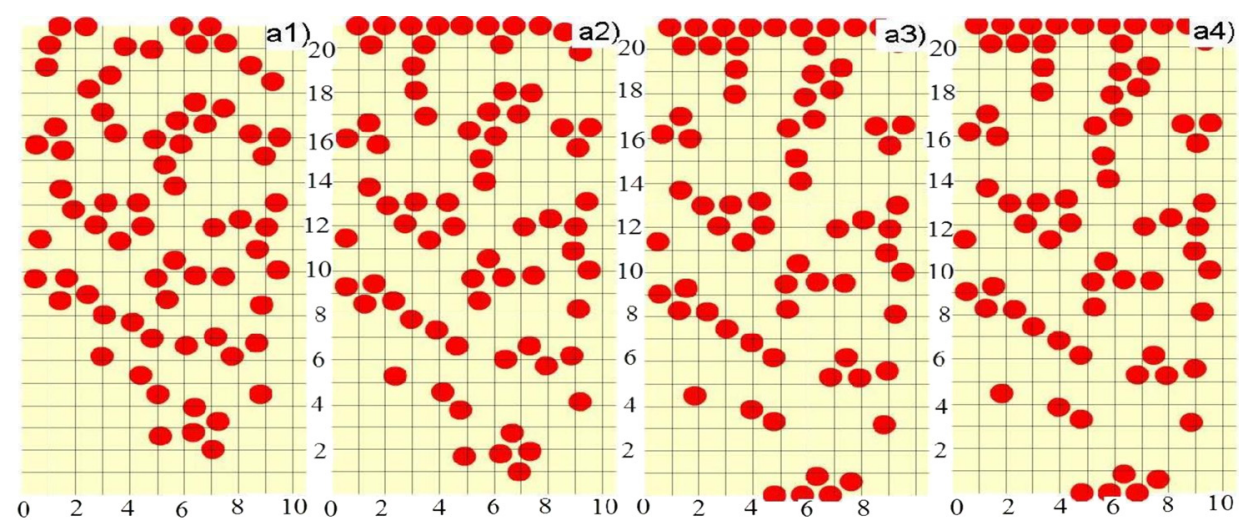

FIG. 2. (Color online) Vacancy dynamics in different crystal fields (upper panels (a): $l=0.2$, lower panels (b): $l=2$ ) at different times after the electric pulse has been applied: $\tau=\tau_{0} \quad[\mathrm{a}(1), \mathrm{b}(1)]$, $\tau=2 \tau_{0} \quad[\mathrm{a}(2), \mathrm{b}(2)], \quad \tau=3 \tau_{0} \quad[\mathrm{a}(3), \mathrm{b}(3)]$,
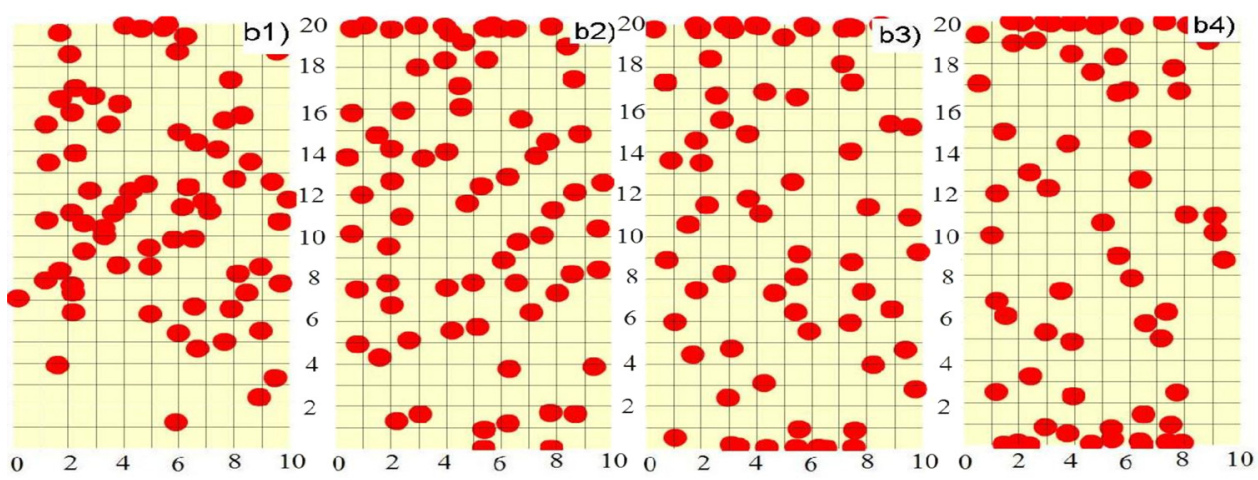
and $\tau=4 \tau_{0}[\mathrm{a}(4), \mathrm{b}(4)]$.

where $x_{\mathrm{i}}^{\alpha}$ is the $\alpha$-coordinate of the $i$ th vacancy $(\alpha=x, y, z), \eta$ is the friction coefficient, $F_{\mathrm{i}}^{\alpha}$ is the $\alpha$-component of electric pulse force, and $\xi^{i}$ is a random force.

The $\mathrm{O}^{2-}$ vacancy-vacancy interaction potential, which is responsible for the clustering, is often modeled as ${ }^{8}$

$$
W(R)=A \exp \left(-R / a_{0}\right)-B / R^{6}+e^{2} /\left(\pi \epsilon_{0} \in R\right),
$$

where the short-range repulsive and attractive parts are represented with the parameters $A=22764.0 \mathrm{eV}, a_{0}=0.01490$ $\mathrm{nm}$, and $B=27.88 \times 10^{-6} \mathrm{eV} \mathrm{nm}{ }^{6}$ in $\mathrm{TiO}_{2-x}$ (Ref. 9), and the long-range Coulomb repulsion is given by the last term, see Fig. 1. Since we simulate a limited number of vacancies, one can refer to each particle in our simulations as a cluster of vacancies, where a cluster-cluster interaction is more conveniently described by the combination of the Lennard-Jones and Coulomb potentials acting with the force

$$
F(R)=\frac{1}{R}\left\{12 E_{L J}\left[\left(R_{\min } / R\right)^{12}-\left(R_{\min } / R\right)^{6}\right]+E_{c} R_{\min } / R\right\},
$$

where the relative strength of the Coulomb potential is given by $E_{c} / E_{L J}=2$. This results in the position of the potential maxima $R_{\max } \approx 2 R_{\min }$ and the height of the potential barrier on the order of the depth of the potential well. The electric pulse strength is taken such that the Coulomb force is about ten times stronger than the maximum attracting force between vacancies, and the interactions are cut-off at a distance of about $R_{\min } / 20$. The Lennard-Jones potential provides somewhat stiffer repulsion at small distances, but otherwise it fairly fits Eq. (2) with the parameters $E_{L J} \approx 2.16 \mathrm{eV}$ and $R_{\min } \approx 0.125 \mathrm{~nm}$ (Fig. 1).

The vacancy distribution depends on the boundary conditions (BCs), the sample size, temperature gradients, ${ }^{2}$ and,

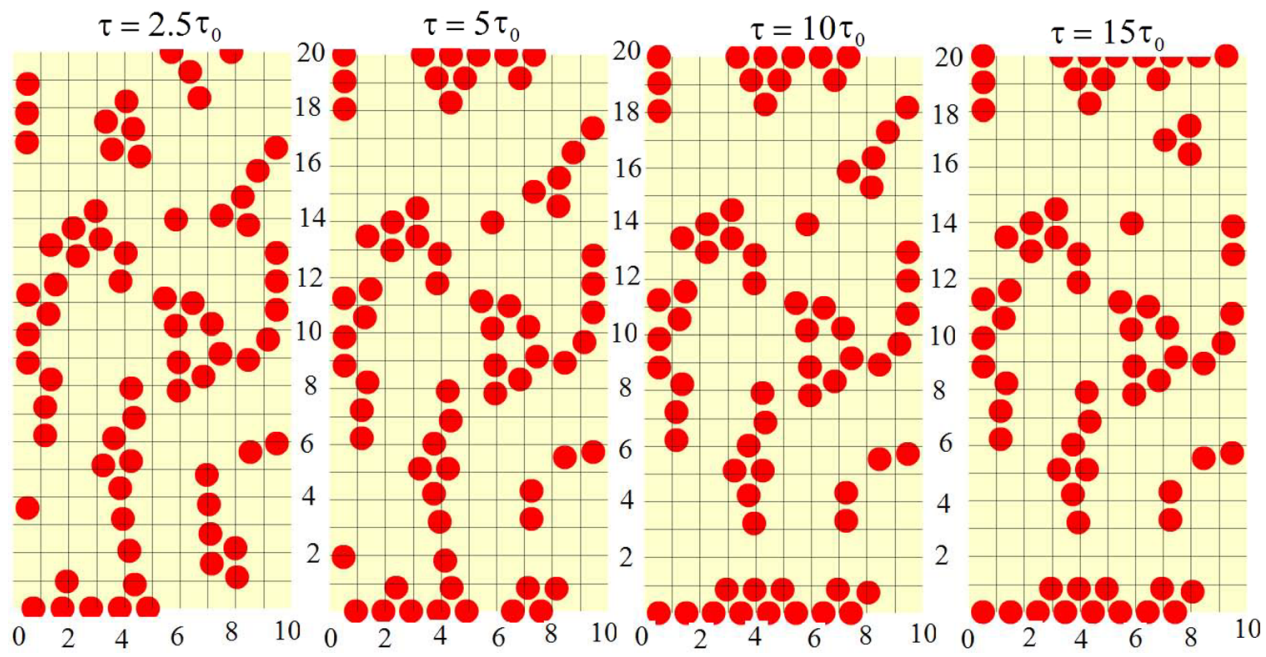

FIG. 3. (Color online) Vacancy dynamics in the crystal field with $l=0.2$ and with sufficiently low temperature after pulse is over. The evolution of vacancies show a very slow (virtually none) dynamics well after the electric pulse has been applied since the crystal field barriers are high with respect to the thermal noise. 
in the present simulations, on the ratio of the lattice constant $a$ and the characteristic scale of the interaction potential $R_{\text {min }}$. Hence, it is most convenient to measure coordinates in units of $R_{\text {min }}$ by introducing $\mathbf{r}=\mathbf{x} / R_{\text {min }}$ while we measure time in units of $t_{0}=R_{\text {min }}{ }^{2} / D$ by introducing $\tau=t / t_{0}$, where $D=k_{B} T / \eta$ is the diffusion coefficient. The Langevin equations in this dimensionless $(\mathbf{r}, \tau)$ space takes the following form:

$$
\begin{aligned}
\frac{d r_{i}^{\alpha}}{d \tau}= & -\frac{\partial\left[V\left(\boldsymbol{r}_{i}\right)+U\left(\boldsymbol{r}_{i}\right)\right] /\left(k_{B} T\right)}{\partial r_{i}^{\alpha}} \\
& -\sum_{j \neq i} \frac{\partial W\left(\boldsymbol{r}_{i}-\boldsymbol{r}_{j}\right) /\left(k_{B} T\right)}{\partial r_{i}^{\alpha}}+\sqrt{2} \xi_{i}^{\alpha}(\tau),
\end{aligned}
$$

where $V\left(\boldsymbol{r}_{i}\right)$ is the electric-field potential, and the components of the (dimensionless) random force, $\xi_{i}^{\alpha}$, satisfies the fluctuation-dissipation relation $\left\langle\xi_{i}^{\alpha}(0) \xi_{j}^{\beta}(\tau)\right\rangle=\delta(\tau) \delta_{\alpha, \beta} \delta_{i, j}$. While the diffusion in $\mathrm{TiO}_{2}$ is anisotropic, for simplicity sake here and below we consider the uniform temperature across the device and the isotropic diffusion of vacancies in a two dimensional (2D) square lattice with the crystal field,

$$
U(\mathbf{r})=U_{0} \sin (2 \pi x / l) \sin (2 \pi y / l),
$$

where $x, y$ are two components of $\mathbf{r}$, and $l=a / R_{\text {min }}$.

We simulate Eq. (4) for $N=70$ vacancies with $l=2$, $U_{0}=4 E_{L J} / 3$ and with $l=0.2, U_{0}=E_{L J} / 6$, respectively, so that the forces $\left(\sim U_{0} / a\right)$ are of the same order of magnitude in both cases. We place all the vacancies randomly near the bottom of a toy sample and then let them evolve according to Eq. (4) inside a rectangular box, which mimics the actual sample. We use the 2D simulation area with the aspect ratio $L_{y} / L_{x}=2$ and periodic BCs along the $x$-direction. Note that the periodic $\mathrm{BC}$ allows us to simulate an infinite area sample using a rather small number of particles. To use periodic BC, we include periodic images of the vacancies with respect to vertical boundaries of the simulation box. We also incorporate opposite polarity charges by adding mirror images of vacancies with respect to the top and the bottom of the sample.

The results of simulations with the homogeneous temperature $\left[k_{B} T \approx 1 / 30\right.$ of the interaction potential well, Fig. 1] are presented in Fig. 2. Different columns refer to different moments in time [time intervals are shown relative to the rectangular electric pulse duration, $\left.\tau_{0}\right]$. The magnitude of the dimensionless electric pulse force is taken as $L_{y} / 2 \tau_{0}$, so that vacancies are pushed by the pulse to the center of the sample. The distribution is spread out and its maximum moves towards the top of the sample with a constant velocity.

The important effect of the crystal field is that clusters appear more ordered than those without the crystal field. ${ }^{2}$ Namely, there are signatures of an orientational order at the angle of $45^{\circ}$ for $l=0.2$ and of spatial voids in the vacancy distribution on the order of a few lattice constants for $l=2$. This indicates that a large variety of different vacancy phases (from liquid-like via smectic or nematic liquid crystal-like to crystal-like phases) can be achieved in proper domains of parameters (such as temperature, vacancy concentration, vacancy pinning, electric pulse intensity, sample shape, etc.) in this strongly interacting system with competing thermal and quenched disorders. Actually, our MD simulations may shed a light on what kind of microscopic distributions could be behind a so-called "random circuit breaker network" behavior phenomenologically introduced in Ref. 10 used to explain both bipolar and unipolar resistance switching in the $\mathrm{Pt} /$ $\mathrm{SrTiO}_{x} / \mathrm{Pt}$ capacitor and changeover between the two regimes.

Here, it is worth mentioning an analogy between vacancies in memristors and superconducting vortices, since in both cases long-range interactions, which can be both repulsive and attractive, and thermal/quenched disorder play a crucial role. It is well known ${ }^{11}$ that a huge variety of vortex phases exists in superconductors. In addition to the standard vortex crystal and vortex liquid phases, different types of smectic phases were predicted and observed in superconductors.

We observe similar behavior in our simulations. Indeed, as observed in our earlier MDs without the crystal field, ${ }^{2}$ the vacancy distribution front keeps moving towards the top of the sample gradually forming filamentary clusters even after switching the voltage pulse off. If the vacancies continue to move for long times after the end of the pulse, it would be difficult to build a non-volatile device. These earlier simulations might be relevant for other systems, like $\mathrm{Li}^{+}, \mathrm{Na}^{+}$, or even $\mathrm{Ag}^{+}$ions in silicon or germanium. In these systems, it is known that the ions continue to move after the pulse is over. Remarkably, we observe in the present MDs that the crystal field stabilizes the clusters after they are formed by lowering the mobility of vacancies. These non-equilibrium metastable vacancy states relax extremely slowly, since they have to overcome crystal field barriers to relax to states having lower energy. Thus, vacancies in memristors can be frozen into a metastable phase for an extremely long time, in analogy to vortices staying in non-equilibrium critical states for many years. As a result, we see a nonvolatile behavior where the vacancies freeze right after the pulse if the crystal field is large enough, as illustrated in Fig. 3, representing the vacancy distributions on a long time scale.

We conclude that manipulating the vacancy dynamics with the crystal field and the vacancy-vacancy interactions could provide a rather rich distribution of phases. We observe a tendency towards crystallization of the vacancies, in agreement with the experimental devices, where the $\mathrm{TiO}_{2}$ material in the switching channels is crystalline. ${ }^{3}$ Our MD simulations indicate that some crystal field potentials provide the orientational ordering and stabilization of electrically conducting channels in memristors that are vital for their nonvolatile memory property. Further 3D MDs of real memristors with the number of oxygen vacancies as large as 1000 and above should provide a powerful approach for understanding and improving these devices.

${ }^{1}$ D. B. Strukov et al., Nature 453, 80 (2008).

${ }^{2}$ S. E. Savel'ev et al., Appl. Phys. A 102, 891 (2011); Nanotechnology 22, 254011 (2011).

${ }^{3}$ J. P. Strachan et al., Adv. Mater. 22, 3573 (2010).

${ }^{4}$ J. Borghetti et al., J. Appl. Phys. 106, 124504 (2009).

${ }^{5}$ S. B. Lee et al., Appl. Phys. Lett. 93, 252102 (2008).

${ }^{6}$ H. Miyaoka et al., Solid State Commun. 123, 399 (2002); K. Tsunoda et al., Appl. Phys. Lett. 90, 113501 (2007); K. M. Kim et al., Appl. Phys. Lett. 90, 242906 (2007); J. R. Jameson et al., Appl. Phys. Lett. 91, 112101 (2007); H. Shima, Appl. Phys. Lett. 94, 082905 (2009); N. Zhong et al., Appl. Phys. Lett. 96, 042107 (2010).

${ }^{7}$ D.-H. Kwon et al., Nat. Nanotechnol. 5, 148 (2010).

${ }^{8}$ C. Meis and J. L. Fleche, Solid State Ionics 101, 333 (1997).

${ }^{9}$ M. Radecka et al., Solid State Ionics 119, 55 (1999).

${ }^{10}$ S. B. Lee et al., Appl. Phys. Lett. 98, 033502 (2011).

${ }^{11}$ G. Blatter et al., Rev Mod. Phys. 66, 1225 (1994). 